\title{
Behaviour of Comet 21P/Giacobini-Zinner during the 1998 perihelion
}

\author{
L.-M. Lara ${ }^{1}$, J. Licandro ${ }^{2}$, A. Oscoz ${ }^{3}$, and V. Motta ${ }^{3,4}$ \\ 1 Instituto de Astrofísica de Andalucía, CSIC, Camino Bajo de Huétor 24, 18008 Granada, Spain \\ e-mail: lara@iaa.es \\ 2 Centro Galileo Galilei \& Telescopio Nazionale Galileo PO Box 565, 38700 Santa Cruz de La Palma, Tenerife, Spain \\ e-mail: licandro@tng.iac.es \\ 3 Instituto de Astrofísica de Canarias, 38205 La Laguna, Tenerife, Spain \\ e-mail: aoscoz@ll.iac.es \\ ${ }^{4}$ Departamento de Astronomía, Facultad de Ciencias, Universidad de la República, Iguá 4225, 11400 Montevideo, Uruguay \\ e-mail: vmotta@ll.iac.es
}

Received 4 March 2002 / Accepted 7 November 2002

\begin{abstract}
Comet 21P/Giacobini-Zinner was observed from Nov. 8 to Dec. 10, 1998. Pre- and post-perihelion CCD images of the gas $\left(\mathrm{CN}, \mathrm{C}_{2}\right)$ and dust (green and red continua) coma were obtained with the $82 \mathrm{~cm} \mathrm{IAC-80} \mathrm{telescope} \mathrm{at} \mathrm{Teide} \mathrm{Observatory}$ (Canary Islands, Spain). For $v_{\mathrm{p}}=0.85 r_{\mathrm{H}}^{-0.5}$ and $v_{\mathrm{d}}=1.19 \mathrm{~km} \mathrm{~s}^{-1}$ (i.e. characteristic of HCN being the CN parent species), the $\mathrm{CN}$ column density profiles are best reproduced with parent and daughter lifetimes of the order of 19300 and $256000 \mathrm{~s}$. An equally good reproduction of the observed profiles is achieved by considering that a mixture of nitrogen compounds expanding at $v_{\mathrm{p}}=1 \mathrm{~km} \mathrm{~s}^{-1}$ and with a lifetime of $19000 \mathrm{~s}$ produces $\mathrm{CN}$ with an ejection velocity of $v_{\mathrm{d}}=2.5 \mathrm{~km} \mathrm{~s}^{-1}$ and a lifetime of $174000 \mathrm{~s}$. Fitting the observed $\mathrm{CN}$ profiles with variable velocities and lifetimes, the results indicate that the nature of the $\mathrm{CN}$ precursor in comet 21P/Giacobini-Zinner is still unclear, ruling out $\mathrm{HCN}$ as the only precursor and favouring a mixture of nitrogen compounds. Regarding $\mathrm{C}_{2}$, the derived lifetimes are 35000 and $62000 \mathrm{~s}$, if the parent and daughter velocities are fixed at $\sim 1 \mathrm{~km} \mathrm{~s}^{-1}$. Gas production rates derived by means of the Vectorial modeling with the mentioned above lifetimes and velocities indicate that (i) the comet activity decreases with decreasing $r_{\mathrm{H}}$ (i.e. peak activity is not reached at the perihelion), and (ii) as already known, the comet is typically depleted in $\mathrm{C}_{2}$ with a $\log \frac{Q_{\mathrm{C}_{2}}}{Q_{\mathrm{CN}}} \approx-0.4$. The azimuthally averaged surface brightness profiles of the continuum images are well fitted with $m \geq 1$ in a $\log B-\log \rho$ representation at projected radial distances $(\rho)$ larger than $1000 \mathrm{~km}$. The continuum light scattered from the dust in the coma of comet Giacobini-Zinner is redder than the Sun light on every date from Nov. 8 to 24 , regardless the cometocentric distance. On Nov. 25, there is a sudden change in the dust color, being considerably bluer than the Sun, whereas on Dec. 7 and 8, the dust became much redder than it was before. These color variations do not seem to be related to sudden variations (relative minimum or maximum) in the cometary activity. The gas-to-dust mass ratio is $\sim 1$, but affected by a large uncertainty (about a factor of 2 ) since the comet was not simultaneously imaged in the $\mathrm{OH}$ band, and the $\mathrm{H}_{2} \mathrm{O}$ production rate has been considered from other measurements taken some weeks before ours.
\end{abstract}

Key words. comets: individual: 21P/Giacobini-Zinner - comets: general

\section{Introduction}

Comet $21 \mathrm{P} /$ Giacobini-Zinner (hereafter referred to as $\mathrm{G}-\mathrm{Z}$ ) is a short period comet $(6.6 \mathrm{yr})$, with perihelion at a distance of $1.03 \mathrm{AU}$, belonging to the Jupiter-family and with a relatively stable orbit since it was discovered in 1900. However, it has been known for long that $\mathrm{G}-\mathrm{Z}$ is an unusual comet in several aspects. Its molecular abundances had been noted as anomalous during previous apparitions, when $\mathrm{C}_{2} / \mathrm{CN}$, $\mathrm{C}_{3} / \mathrm{CN}$ and $\mathrm{NH} / \mathrm{CN}$ abundances were much lower than those measured in other comets (Bobrovnikoff 1927; Herbig 1976;
Cochran \& Barker 1987; Schleicher et al. 1987; Konno \& Wickoff 1989; Beaver et al. 1990) and only slightly lower the $\mathrm{CN} / \mathrm{OH}$ abundance. This fact seems to indicate that $\mathrm{C}_{2}, \mathrm{C}_{3}$ and $\mathrm{NH}$ are underabundant in the coma of $\mathrm{G}-\mathrm{Z}$. In their survey of 85 comets, A'Hearn et al. (1995) describe G-Z as the "prototypical carbon-depleted comet", with $\mathrm{C}_{2}$ and $\mathrm{C}_{3}$ abundances relative to $\mathrm{H}_{2} \mathrm{O} \sim 10$ times lower than the values measured in most comets that define the "normal" cometary composition. A'Hearn et al. (1995) hypothesized that this depletion is associated with the formation 
region in the solar nebula, more concisely in a flat transNeptunian disk called the Edgeworth-Kuiper belt (EKb). On the other hand, the quasi-normality in the $\mathrm{CN} / \mathrm{OH}$ abundance leaves open the possibility that other (excluding the $\mathrm{C}_{2}$ and $\mathrm{C}_{3}$ progenitors) carbon-bearing molecules in the nucleus may not be depleted.

This comet, during its 1985 apparition, also showed the peculiarity of peaking in gas production a full month before perihelion (McFadden et al. 1987), yet the visual light curve did not decline until after perihelion. Photometry in the $1-20 \mu \mathrm{m}$ range by Hanner et al. (1992) confirmed that the visual light curve did not follow either the water production rate (which peaked $\sim 30$ days pre-perihelion) or the scattering and emitting cross section of dust in the inner coma. The shape of the light curve had varied from the 1979 to the 1985 apparition, implying that the location of active areas on the nucleus may have changed. Hanner et al. (1992) also concluded that the low abundance of $\mathrm{C}_{2}, \mathrm{C}_{3}, \mathrm{NH}$ and $\mathrm{NH}_{2}$ is not associated with an unusually low dust/gas ratio.

At the time of the 1998 apparition, to our knowledge, G-Z was only observed by Weaver et al. (1999) during October 2529, and by Mumma et al. (2000) during October 2-10 in the IR, and by Biver et al. (1999) in radio-wavelengths from Oct. 26 to 31 . The IR observations by both groups were made using the same instrument CSHELL at the NASA Infrared Telescope Facility, Mauna Kea, Hawaii. Weaver et al. (1999) derived $\mathrm{H}_{2} \mathrm{O}$ and $\mathrm{CH}_{3} \mathrm{OH}$ production rates of $\sim(2-3) \times 10^{28} \mathrm{~s}^{-1}$ and $\sim 2.7 \times$ $10^{26} \mathrm{~s}^{-1}$, respectively. Emissions from $\mathrm{C}_{2} \mathrm{H}_{6}, \mathrm{CO}, \mathrm{HCN}, \mathrm{C}_{2} \mathrm{H}_{2}$ and $\mathrm{H}_{2} \mathrm{CO}$ were also examined by Weaver et al. (1999) but were not clearly detected, it being possible only to set upper limits for their abundances relative to $\mathrm{H}_{2} \mathrm{O}$. From observations taken a few days before October 25-29, Mumma et al. (2000) were able to detect ethane and carbon monoxide for the first time in a Jupiter-family comet (probably originating in the $\mathrm{EKb}$ ). The ethane production rate was $(7.0 \pm 1.5) \times 10^{25} \mathrm{~s}^{-1}$, whereas carbon monoxide was produced at a rate of $(3.28 \pm 0.64) \times 10^{27}$. When compared with two comets probably originating in the giant-planets region (C/1996 B2 - Hyakutake - and C/1995 $\mathrm{O} 1$ - Hale-Bopp), the production rates and abundance ratios from $\mathrm{G}-\mathrm{Z}$ may imply that ethane was depleted in precometary ices from the region of the solar nebula in which it formed in the $\mathrm{EKb}$.

Charged Coupled Device (CCD) imaging and aperture photometry of G-Z were also conducted by Kiselev et al. (2000a,b) on November 20, 1998 and January 25, 1999. For both dates, there is an uncommon behaviour of polarization, i.e. the wavelength gradient of the polarization of its dust is negative. This effect is not caused by the presence of molecular emission in the pass band of the filter, but it may be due to either a large content of organic matter in its dust (Lebedinets 1991), or to an overabundance of large particles as compared to other comets (Hanner et al. 1992).

In this paper, we present pre- and post-perihelion $\mathrm{CCD}$ images of the gas $\left(\mathrm{CN}, \mathrm{C}_{2}\right)$ and dust (green and red continua) coma of Comet 21P/Giacobini-Zinner, obtained with the $82 \mathrm{~cm}$ IAC-80 Telescope at Teide Observatory (Canary Islands, Spain) from November 8 to December 8, 1998. Assuming certain parent species, this data provide us with parent and daughter lifetimes $\left(\tau_{\mathrm{p}}\right.$ and $\left.\tau_{\mathrm{d}}\right)$, and the production rates $Q$ for $\mathrm{CN}$ and $\mathrm{C}_{2}$ from the best fit of the observed profiles with the Festou modeling (Festou 1981). The continuum images are used (i) to derive radial brightness profiles as a function of the projected distance to the nucleus, $\rho$, and to fit them with the law $\rho^{-m}$, and (ii) to obtain the dust production rate from the $\operatorname{Af} \rho$ parameter. The gas and dust production rates enable us to compute $\mathrm{C}_{2} / \mathrm{CN}$ and $\operatorname{Af} \rho / \mathrm{CN}$ (and thus the gas-todust mass ratio) abundances to better understand the nature of this comet in the framework of our and other previous and/or simultaneous observations.

\section{Data acquisition and reduction}

Comet $\mathrm{G}-\mathrm{Z}$ was observed on nine photometric nights from November 8 to December 8,1998 , using the $82 \mathrm{~cm}$ IAC-80 Telescope at Teide Observatory (Canary Islands, Spain). CCD images were obtained using the Thomson $1024 \times 1024 \mathrm{CCD}$ (pixel size 0. .' 4325, FOV $\sim 7^{\prime} \times 7^{\prime}$ ) and the $\mathrm{CN}, \mathrm{C} 2$, GC and RC narrow-band interference filters specifically designed to isolate continuum regions and some of the stronger emission bands in cometary spectra (see Farnham et al. 2000 for a detailed description of the filters). Several images of the comet, with the telescope tracking on the comet proper motion, were obtained each night using each filter. Details of the observations are presented in Table 1.

Several spectrophotometric standard stars and solar analogs from the list of Farnham et al. (2000) were observed each night at different airmasses to render the absolute calibration of the images. The air-mass coefficient, $k_{\lambda}$, was also determined for each filter, each night, using these data (see Table 2). The $k_{\lambda}$ obtained for the narrowband filters are coherent with the ones obtained for the broad-band filters usually installed at the IAC-80 telescope (see http://www.iac.es/telescopes/ten.html). Also, the day-to-day variations of the extinction coefficients so determined are similar to that observed with the broad-band filters and are mostly due to the variation of the amount of dust from the Sahara desert in the atmosphere of the observatory. As an example, on Nov. 8 there was a lot of dust over the observatory and the $k_{\lambda}$ were larger than usual.

Images were reduced by making the overscan correction, and then using very high $S / N$ ratio sky flat-fields obtained each night in order to correct pixel-to-pixel variations to below $1 \%$. After that, all comet images were divided by their exposure times (in seconds) to normalize the image intensities to counts per second. The contribution of the sky was determined by computing the median of the pixels close to the border of the images, since the comet did not fill the field of view, and it was subtracted from each image. Then, the images were flux calibrated in the HB system, and the underlying continuum and gas contamination were removed following the procedure presented in Farnham et al. (2000).

To obtain the final images of the comet, the position of the comet optocenter in the calibrated images was determined by fitting a two-dimensional Gaussian to the inermost 20 pixels of the coma, all images were recentered and those taken with the same filter on each night were median combined. Examples 
Table 1. Details of the observations.

\begin{tabular}{|c|c|c|c|c|c|c|c|c|c|}
\hline $\begin{array}{c}\text { Date } \\
\text { (1998) }\end{array}$ & UT range & Images & $\begin{array}{c}\text { Total } \\
\text { Exp. } \\
\text { time (s) }\end{array}$ & Filter & $\begin{array}{c}\text { Air } \\
\text { mass } \\
\text { range }\end{array}$ & $\begin{array}{c}\Delta \\
(\mathrm{AU})\end{array}$ & $\begin{array}{c}r_{\mathrm{H}} \\
(\mathrm{AU})\end{array}$ & $\begin{array}{l}\text { Phase } \\
\text { (deg.) }\end{array}$ & $\begin{array}{l}\text { Pixel } \\
\text { scale } \\
(\mathrm{km})\end{array}$ \\
\hline Nov. 8 & $19: 10-21: 02$ & 8 & 2400 & $\mathrm{CN}$ & $1.5-2.2$ & 0.886 & 1.050 & 60.9 & 278 \\
\hline Nov. 8 & $19: 22-20: 45$ & 4 & 1200 & $\mathrm{C} 2$ & $1.4-2.0$ & 0.886 & 1.050 & 60.9 & \\
\hline Nov. 8 & $19: 33-20: 56$ & 4 & 1200 & GC & $1.5-2.1$ & 0.886 & 1.050 & 60.9 & \\
\hline Nov. 8 & $19: 28-20: 51$ & 4 & 1200 & $\mathrm{RC}$ & $1.5-2.1$ & 0.886 & 1.050 & 60.9 & \\
\hline Nov. 10 & $19: 18-20: 58$ & 9 & 2700 & $\mathrm{CN}$ & $1.4-2.2$ & 0.879 & 1.046 & 61.3 & 276 \\
\hline Nov. 10 & $19: 31-20: 44$ & 3 & 900 & $\mathrm{C} 2$ & $1.5-2.0$ & 0.879 & 1.046 & 61.3 & \\
\hline Nov. 10 & $19: 04-20: 21$ & 4 & 1200 & $\mathrm{GC}$ & $1.4-1.8$ & 0.879 & 1.046 & 61.3 & \\
\hline Nov. 10 & $19: 37-20: 50$ & 3 & 900 & $\mathrm{RC}$ & $1.5-2.1$ & 0.879 & 1.046 & 61.3 & \\
\hline Nov. 21 & $19: 59-21: 04$ & 6 & 1800 & $\mathrm{CN}$ & $1.7-2.4$ & 0.853 & 1.034 & 62.3 & 267 \\
\hline Nov. 21 & $20: 16-20: 47$ & 2 & 600 & $\mathrm{C} 2$ & $1.5-2.0$ & 0.853 & 1.034 & 62.3 & \\
\hline Nov. 21 & $20: 58-21: 03$ & 1 & 300 & GC & $2.3-2.3$ & 0.853 & 1.034 & 62.3 & \\
\hline Nov. 21 & $20: 22-20: 53$ & 2 & 600 & $\mathrm{RC}$ & $1.9-2.2$ & 0.853 & 1.034 & 62.3 & \\
\hline Nov. 22 & $18: 58-20: 47$ & 9 & 2700 & $\mathrm{CN}$ & $1.5-2.2$ & 0.851 & 1.034 & 62.3 & 267 \\
\hline Nov. 22 & $19: 19-20: 27$ & 3 & 900 & $\mathrm{C} 2$ & $1.5-2.0$ & 0.851 & 1.034 & 62.3 & \\
\hline Nov. 22 & $19: 39-20: 39$ & 3 & 900 & GC & $1.6-2.1$ & 0.851 & 1.034 & 62.3 & \\
\hline Nov. 22 & $19: 26-20: 33$ & 3 & 900 & $\mathrm{RC}$ & $1.6-2.0$ & 0.851 & 1.034 & 62.3 & \\
\hline Nov. 23 & $18: 59-20: 23$ & 9 & 2700 & $\mathrm{CN}$ & $1.5-1.9$ & 0.852 & 1.034 & 62.3 & 267 \\
\hline Nov. 23 & $19: 19-20: 27$ & 3 & 900 & $\mathrm{C} 2$ & $1.5-2.0$ & 0.852 & 1.034 & 62.3 & \\
\hline Nov. 23 & $19: 27-20: 36$ & 3 & 900 & GC & $1.6-2.1$ & 0.852 & 1.034 & 62.3 & \\
\hline Nov. 23 & $19: 30-20: 47$ & 3 & 900 & $\mathrm{RC}$ & $1.6-2.0$ & 0.852 & 1.034 & 62.3 & \\
\hline Nov. 24 & 18:59-20:19 & 9 & 2700 & $\mathrm{CN}$ & $1.5-1.9$ & 0.851 & 1.035 & 62.2 & 267 \\
\hline Nov. 24 & $19: 16-20: 30$ & 3 & 900 & $\mathrm{C} 2$ & $1.5-2.0$ & 0.851 & 1.035 & 62.2 & \\
\hline Nov. 24 & $19: 24-20: 38$ & 3 & 900 & GC & $1.6-2.1$ & 0.851 & 1.035 & 62.2 & \\
\hline Nov. 24 & $19: 30-20: 33$ & 3 & 900 & $\mathrm{RC}$ & $1.6-2.0$ & 0.851 & 1.035 & 62.2 & \\
\hline Nov. 25 & $19: 01-20: 22$ & 9 & 2700 & $\mathrm{CN}$ & $1.5-1.9$ & 0.850 & 1.035 & 62.2 & 267 \\
\hline Nov. 25 & $19: 18-20: 28$ & 3 & 900 & $\mathrm{C} 2$ & $1.6-2.0$ & 0.850 & 1.035 & 62.2 & \\
\hline Nov. 25 & $19: 30-20: 39$ & 3 & 900 & $\mathrm{GC}$ & $1.6-2.1$ & 0.850 & 1.035 & 62.2 & \\
\hline Nov. 25 & $19: 24-20: 33$ & 3 & 900 & $\mathrm{RC}$ & $1.6-2.0$ & 0.850 & 1.035 & 62.2 & \\
\hline Dec. 7 & $19: 06-21: 03$ & 8 & 2400 & $\mathrm{CN}$ & $1.6-2.4$ & 0.863 & 1.058 & 60.6 & 271 \\
\hline Dec. 7 & $19: 38-20: 30$ & 3 & 900 & $\mathrm{C} 2$ & $1.7-2.1$ & 0.863 & 1.058 & 60.6 & \\
\hline Dec. 7 & $20: 03-20: 57$ & 4 & 1200 & GC & $1.8-2.3$ & 0.863 & 1.058 & 60.6 & \\
\hline Dec. 7 & $19: 50-20: 51$ & 4 & 1200 & $\mathrm{RC}$ & $1.8-2.0$ & 0.863 & 1.058 & 60.6 & \\
\hline Dec. 8 & 19:02-20:40 & 7 & 2100 & $\mathrm{CN}$ & $1.6-2.2$ & 0.866 & 1.061 & 60.3 & 271 \\
\hline Dec. 8 & $19: 30-20: 46$ & 4 & 1200 & $\mathrm{C} 2$ & $1.7-2.2$ & 0.866 & 1.061 & 60.3 & \\
\hline Dec. 8 & $19: 55-20: 19$ & 3 & 900 & GC & $1.8-2.0$ & 0.866 & 1.061 & 60.3 & \\
\hline Dec. 8 & $19: 50-21: 01$ & 3 & 900 & $\mathrm{RC}$ & $1.7-2.4$ & 0.866 & 1.061 & 60.3 & \\
\hline
\end{tabular}

Images: number of images taken; Phase, $r_{\mathrm{H}}, \Delta$ : the phase angle, heliocentric, and geocentric distances of the comet during the observations, respectively.

of the final images taken on Nov. 10 are shown in Fig. 1 in a logarithmic look-up table. We should note that, as we did not observe the comet in the $C_{3}$ band, it was impossible to remove any possible $C_{3}$ contamination in the $C N$ band. In any case, this contamination should be very small $(<10 \%)$ in the particular case of $\mathrm{G}-\mathrm{Z}$ as it is a carbon-depleted comet.

\section{Results}

\subsection{Spatial gas profiles}

All the images were used to investigate the $\mathrm{CN}$ and $\mathrm{C}_{2}$ profiles derived from an azimuthal average, leading to energy flux as a function of the projected radial distance, $\rho$. The conversion of the emission band fluxes into column densities was done with a constant $g$-factor for $\mathrm{C}_{2}$ equal to $4.476 \times 10^{-13} \mathrm{ergs} \mathrm{s}^{-1} \mathrm{~mol}^{-1}$
Table 2. Air-mass coefficients.

\begin{tabular}{lcccc}
\hline \hline Date (1998) & $k_{\mathrm{CN}}$ & $k_{\mathrm{C} 2}$ & $k_{\mathrm{GC}}$ & $k_{\mathrm{RC}}$ \\
\hline Nov. 8 & 0.50 & 0.25 & 0.27 & 0.12 \\
Nov. 10 & 0.38 & 0.11 & 0.13 & 0.04 \\
Nov. 21 & 0.47 & 0.12 & 0.13 & 0.05 \\
Nov. 22 & 0.48 & 0.06 & 0.09 & 0.02 \\
Nov. 23 & 0.35 & 0.06 & 0.08 & 0.01 \\
Nov. 24 & 0.45 & 0.19 & 0.14 & 0.06 \\
Nov. 25 & 0.44 & 0.08 & 0.09 & 0.01 \\
Dec. 7 & 0.38 & 0.16 & 0.11 & 0.06 \\
Dec. 8 & 0.33 & 0.09 & 0.12 & 0.02 \\
\hline
\end{tabular}

(A'Hearn et al. 1985) and $g$-factors for $\mathrm{CN}$ that were calculated for the heliocentric distances and heliocentric velocities of Comet 21P/Giacobini-Zinner from Nov. 8 to Dec. 8, 1998 


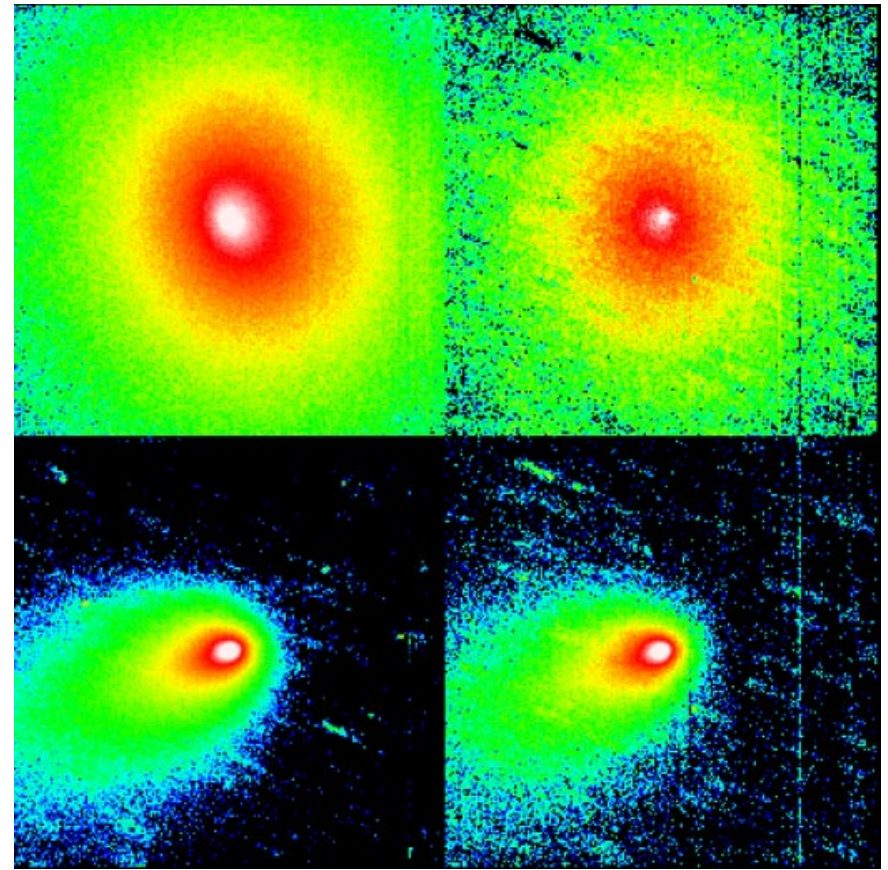

Fig. 1. Series of bias and flat-field corrected images taken on Nov. 10, 1998. After that images were flux calibrated in the HB system, and the underlying continuum and gas contamination were removed. From left to right and top to bottom: $\mathrm{CN}, \mathrm{C}_{2}$, red continuum, and green continuum. The scale is 173 ." $43 \times 173$." 43 (i.e. $111478 \times 111478 \mathrm{~km}$ at the comet distance) for all of them. North is down and East to the left. The $\mathrm{CN}$ image shows a clear elongation in the direction perpendicular to the Sun position, as projected on the plane of the sky. $\mathrm{C}_{2}$, green and red continuum images show the typical spherical gas coma, and the dust coma and tail, respectively.

from the set of values given by Schleicher (1983), and are listed in Table 3.

The azimuthally averaged $\mathrm{CN}$ and $\mathrm{C}_{2}$ spatial profiles are presented in Figs. 2 and 3, respectively, together with the theoretical profiles best resembling the observed ones. These theoretical profiles were obtained by means of the Festou modeling (Festou 1981), where parent and daughter and lifetimes $\left(\tau_{\mathrm{p}}, \tau_{\mathrm{d}}\right)$ at $r_{\mathrm{H}}=1 \mathrm{AU}$, as well as production rates, $Q$, were derived as follows.

Assuming that $\mathrm{HCN}$ might be the $\mathrm{CN}$ precursor, we fix $v_{\mathrm{p}}=0.85 \times r_{\mathrm{H}}^{-0.5}\left(\right.$ Biver et al. 1999b, 2000) and $v_{\mathrm{d}}=1.19 \mathrm{~km} \mathrm{~s}^{-1}$ (Bockelée-Morvan \& Crovisier 1985). To derive the $\left(\tau_{\mathrm{p}}, \tau_{\mathrm{d}}\right)$ that best resemble the shape of the observed profiles, we constructed theoretical radial profiles with an arbitrary (however approximated) gas production rate with lifetimes spanning the reported values, i.e. $\tau_{\mathrm{p}}$ ranging from $1.6 \times 10^{4} \mathrm{~s}$ to $6.6 \times 10^{4} \mathrm{~s}$, whereas $\tau_{\mathrm{d}}$ covered the $(1.5-4.0) \times 10^{5} \mathrm{~s}$. Once the theoretical profile was computed, we compared it with the observed profile by dividing both profiles. We selected the profiles computed with $\left(\tau_{\mathrm{p}}, \tau_{\mathrm{d}}\right)$ giving rise to those theoretical profiles whose standard deviation of the quotient calculated at distances $3<\log \rho<4.64$ (i.e. between $1 \times 10^{3}$ and $3.2 \times 10^{4} \mathrm{~km}$ not to hit the sky) was lower than $3 \times 10^{-3}$. All of the $\left(\tau_{\mathrm{p}}, \tau_{\mathrm{d}}\right)$ better fitting the observed profiles for every date (i.e. parameters producing quotient images with the lowest standard deviation, in
CN

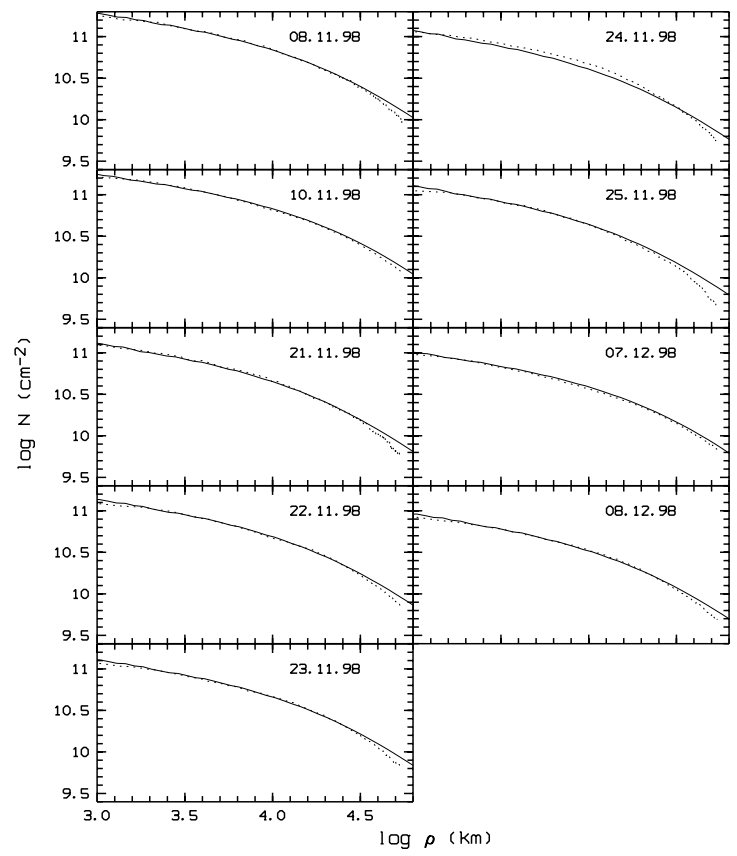

Fig. 2. Azimuthally averaged $\mathrm{CN}$ column density profiles of Comet 21P/Giacobini-Zinner (dots). The solid lines represent the results obtained by means of the Vectorial model with fixed $v_{\mathrm{p}}=0.85 \times r_{\mathrm{H}}^{-0.5}$ and $v_{\mathrm{d}}=1.19 \mathrm{~km} \mathrm{~s}^{-1}$ whereas $\left(\tau_{\mathrm{p}}, \tau_{\mathrm{d}}\right)$ and $Q_{\mathrm{CN}}$ are the best fit parameters listed in Table 3 under these velocities conditions.

some cases as low as $3.6 \times 10^{-4}$ ) were then averaged to produce the values listed in Table 3 . These daily averages have standard deviations $\leq 17 \%$ for $\tau_{\mathrm{p}}$, and $\leq 24 \%$ for $\tau_{\mathrm{d}}$. The gas production rates were obtained once the lifetimes were derived and the values are also presented in Table 3. As the $\mathrm{CN}$ precursor is not clearly known, according to Krasnopolsky (1991) a mixture of $\mathrm{C}_{4} \mathrm{~N}_{2}$ and $\mathrm{HC}_{3} \mathrm{~N}$ expanding at $1 \mathrm{~km} \mathrm{~s}^{-1}$, might give rise to $\mathrm{CN}$ with an ejection velocity of $2.5 \mathrm{~km} \mathrm{~s}^{-1}$. By considering these velocities, the procedure to derive lifetimes was the same as outlined above and the best theoretical profiles were obtained with the lifetimes listed in parenthesis in Table 3 where the uncertainties in both lifetimes are of the same order as in the previous case. Differences among individual $\tau_{\mathrm{p}}$ and $\tau_{\mathrm{d}}$ are not outstandingly large. As a final test, we additionally allowed $\left(v_{\mathrm{p}}, v_{\mathrm{d}}\right)$ to vary from 0.6 to $1.2 \mathrm{~km} \mathrm{~s}^{-1}$ and 1.0 to $3.0 \mathrm{~km} \mathrm{~s}^{-1}$, respectively. Theoretical profiles were obtained for every combination of $\left(v_{\mathrm{p}}, v_{\mathrm{d}}, \tau_{\mathrm{p}}, \tau_{\mathrm{d}}\right)$ and then compared to the observed ones following the same criteria as previously mentioned. In this case, the values of $\left(v_{\mathrm{p}}, v_{\mathrm{d}}, \tau_{\mathrm{p}}, \tau_{\mathrm{d}}\right)$ providing the best daily fits are affected by an uncertainty of $\leq 25 \%$. Averaging these pre- and post-perihelion values, we obtain $v_{\mathrm{p}}=(0.84 \pm 0.06) \mathrm{km} \mathrm{s}^{-1}$, $v_{\mathrm{d}}=(1.38 \pm 0.37) \mathrm{km} \mathrm{s}^{-1}, \tau_{\mathrm{p}}=(19.5 \pm 2.2) \times 10^{3} \mathrm{~s}$ and $\tau_{\mathrm{d}}=(2.68 \pm 0.18) \times 10^{5} \mathrm{~s}$, where the errors represent the standard deviation of the sample. It should be noted that the parent and daughter velocities so derived are merely indicative. The profiles are very sensitive to the parent and daughter scale lengths, and therefore to the two products $v_{\mathrm{p}} \tau_{\mathrm{p}}$ and $v_{\mathrm{d}} \tau_{\mathrm{d}}$ of the Haser-equivalent parameters (Combi \& Delsemme 1980a,b) or to more complex combinations of the physical parameters. 
Table 3. $\mathrm{CN} g$-factors, $\tau_{\mathrm{p}}, \tau_{\mathrm{d}}$ and $Q$ derived from the best fits to the observed profile by using the Festou modeling assuming that $v_{\mathrm{p}}=0.85 \times r_{\mathrm{H}}^{-0.5}$ and $v_{\mathrm{d}}=1.19 \mathrm{~km} \mathrm{~s}^{-1}$ or that $v_{\mathrm{p}}=1 \mathrm{~km} \mathrm{~s}^{-1}$ and $v_{\mathrm{d}}=2.5 \mathrm{~km} \mathrm{~s}^{-1}$ (values in parenthesis). CN production rate from Haser modeling assuming standard $l_{\mathrm{p}}$ and $l_{\mathrm{d}}$. Measurement of the dust-to-gas ratio by means of $\log \frac{A f \rho}{Q_{\mathrm{CN}}}$.

\begin{tabular}{|c|c|c|c|c|c|c|}
\hline $\begin{array}{l}\text { Date } \\
1998\end{array}$ & $\begin{array}{c}g-\text { factor }^{a} \\
10^{-13}\end{array}$ & $\begin{array}{c}\tau_{\mathrm{p}} \\
10^{4} \mathrm{~s}\end{array}$ & $\begin{array}{c}\tau_{\mathrm{d}} \\
10^{5} \mathrm{~s}\end{array}$ & $\begin{array}{c}Q_{\mathrm{Fes}} \\
10^{25} \mathrm{~s}^{-1}\end{array}$ & $\begin{array}{c}Q_{\text {Has }} \\
10^{25} \mathrm{~s}^{-1}\end{array}$ & $\begin{array}{c}{ }^{b} \log \frac{A f \rho}{Q_{\mathrm{CN}}} \\
\mathrm{cm} \mathrm{s}\end{array}$ \\
\hline Nov. 8 & 3.16 & $1.91(1.97)$ & $2.69(1.98)$ & $4.72(9.27)$ & 4.44 & -22.82 \\
\hline Nov. 10 & 3.04 & $2.45(2.21)$ & $2.23(2.08)$ & $5.20(9.59)$ & 4.34 & -22.82 \\
\hline Nov. 21 & 2.36 & $1.74(1.71)$ & $2.65(1.34)$ & $2.95(5.95)$ & 2.87 & -22.83 \\
\hline Nov. 22 & 2.42 & $1.87(1.94)$ & $2.73(1.86)$ & $3.30(6.51)$ & 3.10 & -22.84 \\
\hline Nov. 23 & 2.50 & 1.87 (1.99) & $2.74(1.70)$ & $3.09(6.20)$ & 2.93 & -22.98 \\
\hline Nov. 24 & 2.56 & $1.69(1.80)$ & $2.58(1.68)$ & $2.86(5.70)$ & 2.82 & -22.87 \\
\hline Nov. 25 & 2.70 & $1.71(1.63)$ & $2.63(1.33)$ & $2.66(5.34)$ & 2.67 & -22.94 \\
\hline Dec. 7 & 4.31 & $2.31(2.08)$ & $2.16(2.04)$ & $2.92(5.40)$ & 2.45 & -23.06 \\
\hline Dec. 8 & 4.35 & $1.83(1.81)$ & $2.66(1.65)$ & $2.23(4.43)$ & 2.14 & -23.03 \\
\hline Average $^{c}$ & & $1.93 \pm 0.27$ & $2.56 \pm 0.21$ & $(2.86 \pm 0.34)^{e}$ & $(2.71 \pm 0.32)^{e}$ & $-22.91 \pm 0.09$ \\
\hline Average $^{d}$ & & $1.90 \pm 0.18$ & $1.74 \pm 0.28$ & $(5.64 \pm 0.68)^{e}$ & & \\
\hline
\end{tabular}

${ }^{a} \mathrm{erg} \mathrm{s}^{-1} \mathrm{~mol}^{-1}$.

${ }^{b} \mathrm{CN}$ production rate derived from the Haser modeling, and Af $\rho$ obtained by integrating the green continuum flux in a circular aperture of $\rho<10000 \mathrm{~km}$.

${ }^{c}$ Pre- and post-perihelion averaged values in the case of $v_{\mathrm{p}}=0.85 \times r_{\mathrm{H}}^{-0.5}$ and $v_{\mathrm{d}}=1.19 \mathrm{~km} \mathrm{~s}^{-1}$.

${ }^{d}$ Pre- and post-perihelion averaged values in the case of $v_{\mathrm{p}}=1 \mathrm{~km} \mathrm{~s}^{-1}$ and $v_{\mathrm{d}}=2.5 \mathrm{~km} \mathrm{~s}^{-1}$.

${ }^{e}$ Averaged values by considering postperihelion production rates.

C2

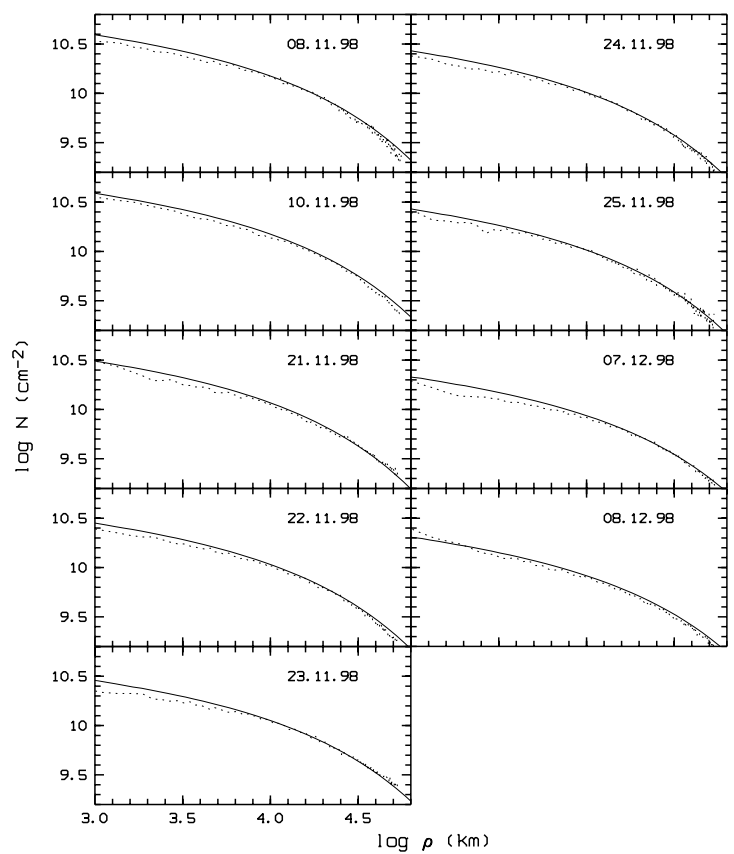

Fig.3. Azimuthally averaged $\mathrm{C} 2$ column density profiles of Comet 21P/Giacobini-Zinner (dots). The solid lines represent the results obtained by means of the Vectorial model with parent and daughter lifetimes and $Q_{\mathrm{C}_{2}}$ listed in Table 4 .

Theoretical predictions of the photodissociation time scales for many cometary species under solar irradiance have been provided by Huebner \& Link (1999) and Huebner et al. (1992). Solar photo rate coefficients and excess energies for dissociation and ionization are calculated both for low and high solar activity. According to Huebner \& Link (1999), the lifetimes for
$\mathrm{HCN}$ and $\mathrm{CN}$ at $1 \mathrm{AU}$ amount to $7.94 \times 10^{4} \mathrm{~s}$ and $3.15 \times 10^{5} \mathrm{~s}$ at low solar activity, and to $3.19 \times 10^{4} \mathrm{~s}$ and $1.34 \times 10^{5} \mathrm{~s}$ at high solar activity. Bockelée-Morvan \& Crovisier (1985) reported the HCN photodissociation rate of the order of $1.5 \times 10^{-5} \mathrm{~s}^{-1}$, i.e. lifetime $\sim 6.7 \times 10^{4} \mathrm{~s}$. A more precise $\mathrm{HCN}$ lifetime, corresponding to the solar activity of Nov.-Dec. 1998, may be estimated as $4.8 \times 10^{4} \mathrm{~s}$ (Crovisier 2002, private communication). From our analysis of the $\mathrm{CN}$ profiles, the parent lifetime we obtain, $(1.93 \pm 0.27) \times 10^{4} \mathrm{~s}$, is lower than that expected for $\mathrm{HCN}$ as the sole precursor of $\mathrm{CN}$. On the other hand, the deduced $\mathrm{CN}$ lifetime, $(2.56 \pm 0.21) \times 10^{5} \mathrm{~s}$, agrees well with theoretical determinations for a moderate-to-high solar activity. For a mixture of nitrogen compounds (for example $\mathrm{C}_{4} \mathrm{~N}_{2}$ and $\mathrm{HC}_{3} \mathrm{~N}$ ) as the $\mathrm{CN}$ parent species, the obtained $\tau_{\mathrm{p}}=(1.90 \pm 0.18) \times 10^{4} \mathrm{~s}$ approaches the theoretical values of cyanoacetylene $(1.4-2.6) \times 10^{4} \mathrm{~s}$ and, to a lesser extent, of dicyanogen, $(0.74-2) \times 10^{4} \mathrm{~s}$. A mixture of nitrogen compounds, richer in $\mathrm{HC}_{3} \mathrm{~N}$ than in $\mathrm{C}_{4} \mathrm{~N}_{2}$, not ruling out $\mathrm{HCN}$, could be considered as the $\mathrm{CN}$ precursor in $\mathrm{G}-\mathrm{Z}$.

The results of the problem of four parameter (velocities and lifetimes) fits do not provide a firm constraint to the "determination" of the $\mathrm{CN}$ parent species. Although $v_{\mathrm{p}}$ favours $\mathrm{HCN}$ (an expansion velocity 10\% lower than that deduced for Comet Hyakutake and Comet Lee can be expected, Biver et al. 1999b, 2000, 2002, private communication), the deduced parent lifetime is too short compared to $4.8 \times 10^{4} \mathrm{~s}$ as deduced for the solar activity of Nov.-Dec. 1998 (Crovisier 2002, private communication). Similarly, a CN ejection velocity of $(1.38 \pm 0.37) \mathrm{km} \mathrm{s}^{-1}$ might rule out $\mathrm{HCN}$ as its only precursor (note that the uncertainty is large and $1.19 \mathrm{~km} \mathrm{~s}^{-1}$ is within the $v_{\mathrm{d}}$ derived values), pointing to a mixture of nitrogen compounds (hydrogen cyanide, cyanoacetylene, dicyanogen) as cyanogen parent species. 
Given that $\mathrm{CN}$ production rates can considerably vary as a function of parent and daughter velocities and lifetimes (see Table 3), we have also used the Haser modeling (Haser 1957) to fit the observed column density profiles in order to consistently compare our results with those published in the 85 comet survey by A'Hearn et al. (1995), or with G-Z specific observations (Schleicher et al. 1987; Beaver et al. 1990; Churyumov et al. 1991; Landaberry et al. 1991; Singh et al. 1997; and Ellis $\&$ Neff 2000). Although the Haser formalism is physically unrealistic, it is an empirical description that can serve as a basis of comparison of gas production and distribution in different comets observed under quite different circumstances of heliocentric and geocentric distances and aperture sizes. The Haser model scale lengths are not meant to be true molecular scale lengths, but rather values that best fit the observed spatial distribution when using this formalism. The resulting production rates can be compared with those derived from the more physically relevant production as obtained from the Vectorial modeling (Festou 1981). Thus, we use the Haser scalelength equal to $1.3 \times 10^{4}$ and $2.1 \times 10^{5} \mathrm{~km}$, for $\mathrm{CN}$ parent and $\mathrm{CN}$ itself, respectively, with a $r_{\mathrm{H}}^{2}$ variation (A'Hearn et al. 1995; Randall et al. 1992). Velocities are held constant at $1 \mathrm{~km} \mathrm{~s}^{-1}$. The best $\mathrm{CN}$ production rates fitting the observed profiles are also included in Table 3. Differences in $Q$ from both models can be as high as a factor of 2 . Fits to observed profiles by means of this formalism are as good as those presented in Fig. 2 with the Vectorial modeling (Festou 1981).

Regarding $\mathrm{C}_{2}$, we followed the same approach as for $\mathrm{CN}$. Parent and daughter lifetimes for the Vectorial modeling ranged from 30000 to $45000 \mathrm{~s}$, and from 40000 to $100000 \mathrm{~s}$, respectively. Velocities were held constant and equal to $1 \mathrm{~km} \mathrm{~s}^{-1}$ (values proven to be a reasonable approximation from the analysis of the coma distortions caused by radiation pressure acceleration, Schulz et al. 1993). It is well known that $C_{2}$ does not seem to be produced by a single photolytic step, the observed profiles being flatter than the ones produced by the vectorial or Haser model in the inner coma. By taking $v_{\mathrm{p}}$ of the same order as $\mathrm{CN}$ (i.e. $v_{\mathrm{p}} \approx 0.83 \mathrm{~km} \mathrm{~s}^{-1}$ ), the $\mathrm{C}_{2}$ parent lifetime has to be larger than $10^{5} \mathrm{~s}$ to obtain a reasonable fit of the $\mathrm{C}_{2}$ column density at distances shorter than $5000 \mathrm{~km}$. After computing theoretical profiles for every combination of $\tau_{\mathrm{p}}$ and $\tau_{\mathrm{d}}$ and comparing them with the observed ones, we averaged those pairs $\left(\tau_{\mathrm{p}}, \tau_{\mathrm{d}}\right)$ satisfying the criterium previously stated. The resulting lifetimes are presented in Table 4 together with the gas production rates computed by means of the Vectorial modeling. Bearing in mind that the $\mathrm{C}_{2}$ parent remains unknown (halo of large icy particles, three-step grandparent-parent-daughter, and $\mathrm{CHON}$ grain halo scenarios have been claimed), the deduced lifetimes are of the same order as determinations for other comets such as Comet Wilson (1987VII) (Schulz et al. 1993). The Haser formalism has also provided us with $\mathrm{C}_{2}$ production rates by considering $l_{\mathrm{p}}=2.2 \times 10^{4} \mathrm{~km}$ and $l_{\mathrm{d}}=6.6 \times 10^{4} \mathrm{~km}$ with a $r_{\mathrm{H}}^{2}$ dependence, $v_{\mathrm{p}}=v_{\mathrm{d}}=1 \mathrm{~km} \mathrm{~s}^{-1}$. As it is the case for $\mathrm{CN}$, gas production rates derived from the Vectorial and from the Haser modeling also differ by as much as $\sim 43 \%$. The last column in Table 4 lists the $\log$ of the $\mathrm{CN} / \mathrm{C}_{2}$ abundance by considering gas production rates computed from the Haser modeling for both species.



Fig. 4. Brightness profiles of Comet 21P/Giacobini-Zinner in the green continuum. The profiles were obtained from azimuthal averaging of the images. The surface brightness, $B$, is plotted against the projected radius, $\rho$, in double logarithmic representation. Dots represent the observed profiles and the solid lines are the best fits verifying $\frac{\mathrm{d}(\log B)}{\mathrm{d}(\log \rho)}=-m$ starting at $\log \rho \geq 3$, with slopes listed in Table 5 .

\subsection{Dust profiles}

The comet was imaged in two different continuum regions centered at $5259 \AA$ and $7133 \AA$, green and red, respectively. The flux calibrated continuum profiles were multiplied by $4 \pi \Delta^{2}$ and $r_{\mathrm{H}}^{2}$ and normalized to a unit area of $1 \mathrm{~km}^{2}$ at the position of the comet. This leads to a surface brightness in erg s $\mathrm{s}^{-1} \mathrm{~km}^{-2}$, which is independent of the geocentric distance $\Delta$ and normalized to the solar flux at $r_{\mathrm{H}}=1 \mathrm{AU}$ (see Schulz et al. 1994). It therefore allows a direct comparison of the day-to-day variations in the continuum images, and thus in the continuum profiles.

Figures 4 and 5 show the brightness profiles derived from an azimuthal average of the continuum images in a log-log representation. The profiles exhibit a normal radially expanding dust coma and they can be well fitted with a $\rho^{-m}$ law with $0.82<m<1.33$ (see Table 5) for $3<\log \rho<4.4$, i.e. between $1 \times 10^{3} \mathrm{~km}$ and $2.5 \times 10^{4} \mathrm{~km}$ projected radial distance from the nucleus. The effect of radiation pressure is clearly visible leading to slopes that are always somewhat higher than 1 . This is consistent with a steady state coma for a constant dust production rate and long-lived grains expanding radially outwards, where the spatial number density should decrease as $r^{-2}, r$ being the nucleocentric radial distance. Consequently, the projected surface brightness would decrease as $\rho^{-1}$.

Estimates of the dust production in comets are usually made by means of the parameter $A(\theta) f \rho$ (A'Hearn et al. 1984). $A(\theta)$ is the Bond albedo for the particular scattering angle, $\theta$, 
Table 4. $\tau_{\mathrm{p}}, \tau_{\mathrm{d}}$ and $Q$ for $\mathrm{C}_{2}$ derived from the best fits to the observed profile by using the Vectorial modeling. $\mathrm{C}_{2}$ production rate from Haser modeling assuming standard $l_{\mathrm{p}}$ and $l_{\mathrm{d}}$. Measurement of the $\mathrm{C}_{2}$ to $\mathrm{CN}$ ratio.

\begin{tabular}{|c|c|c|c|c|c|}
\hline $\begin{array}{l}\text { Date } \\
1998\end{array}$ & $\begin{array}{c}\tau_{\mathrm{p}} \\
10^{3} \mathrm{~s}\end{array}$ & $\begin{array}{c}\tau_{\mathrm{d}} \\
10^{3} \mathrm{~s}\end{array}$ & $\begin{array}{c}Q_{\mathrm{Fes}} \\
10^{25} \mathrm{~s}^{-1}\end{array}$ & $\begin{array}{c}Q_{\text {Has }} \\
10^{25} \mathrm{~s}^{-1}\end{array}$ & ${ }^{a} \log \frac{Q_{\mathrm{C}_{2}}}{Q_{\mathrm{CN}}}$ \\
\hline Nov. 8 & 33.5 & 56.6 & 2.08 & 1.45 & -0.48 \\
\hline Nov. 10 & 33.6 & 61.5 & 2.02 & 1.44 & -0.48 \\
\hline Nov. 21 & 32.5 & 57.9 & 1.56 & 1.13 & -0.40 \\
\hline Nov. 22 & 34.0 & 58.8 & 1.47 & 1.04 & -0.47 \\
\hline Nov. 23 & 36.0 & 67.0 & 1.54 & 1.11 & -0.42 \\
\hline Nov. 24 & 34.4 & 60.1 & 1.41 & 1.00 & -0.45 \\
\hline Nov. 25 & 33.6 & 57.1 & 1.40 & 1.00 & -0.43 \\
\hline Dec. 7 & 37.6 & 70.4 & 1.23 & 0.88 & -0.44 \\
\hline Dec. 8 & 37.6 & 70.4 & 1.18 & 0.84 & -0.41 \\
\hline Average & $34.7 \pm 1.9$ & $62.2 \pm 5.6$ & $(1.44 \pm 0.12)^{b}$ & $(1.00 \pm 0.11)^{b}$ & $-0.44 \pm 0.03$ \\
\hline
\end{tabular}

${ }^{a} \mathrm{CN}$ and $\mathrm{C}_{2}$ Production rates derived from the Haser formalism. ${ }^{b}$ Post-perihelion $Q_{\mathrm{C}_{2}}$ average.

Table 5. Linear fits to the brightness continuum profiles. Dust production rate by means of the parameter $A f \rho$ and color of the cometary grains measured as the reddening of the dust.

\begin{tabular}{lcccccccc}
\hline \hline & \multicolumn{3}{c}{ Green Continuum } & \multicolumn{2}{c}{ Red Continuum } & \multicolumn{2}{c}{ Reddening } \\
\hline Date & $m$ & \multicolumn{2}{c}{$A f \rho(\mathrm{cm})$} & $m$ & \multicolumn{2}{c}{$A f \rho(\mathrm{cm})$} & \multicolumn{2}{c}{$\% / 1000 \AA$} \\
\hline 1998 & \multicolumn{3}{c}{$10^{4} \mathrm{~km}$} & $1.5 \times 10^{4} \mathrm{~km}$ & \multicolumn{1}{c}{$10^{4} \mathrm{~km}$} & $1.5 \times 10^{4} \mathrm{~km}$ & $10^{4} \mathrm{~km}$ & $1.5 \times 10^{4} \mathrm{~km}$ \\
\hline Nov. 8 & 1.26 & 670 & 822 & 1.25 & 830 & 1010 & 12.8 & 12.2 \\
Nov. 10 & 1.26 & 656 & 797 & 1.24 & 644 & 806 & -0.9 & 6.7 \\
Nov. 21 & 1.29 & 420 & 544 & 1.18 & 567 & 649 & 18.7 & 10.3 \\
Nov. 22 & 1.27 & 444 & 548 & 1.22 & 564 & 669 & 14.5 & 11.8 \\
Nov. 23 & 1.12 & 305 & 354 & 1.00 & 388 & 401 & 14.6 & 7.1 \\
Nov. 24 & 1.23 & 380 & 462 & 1.21 & 440 & 522 & 8.4 & 7.1 \\
Nov. 25 & 1.12 & 309 & 353 & 0.82 & 223 & 206 & -14.9 & -22.0 \\
Dec. 7 & 1.26 & 209 & 273 & 1.19 & 300 & 363 & 23.3 & 17.4 \\
Dec. 8 & 1.33 & 197 & 264 & 1.21 & 302 & 367 & 28.5 & 20.6 \\
\hline
\end{tabular}

of the observations, $f$ is the filling factor of the grains in the field of view (number of grains per unit area times their mean cross-section divided by the area of the field of view), and $\rho$ is the radius of the assumed circular field of view. This parameter assumes that physical characteristics of the dust grains remain unchanged as they move outward and that the column of grains is optically thin. Table 5 also contains the values for $A(\theta) f \rho$ computed in circular apertures of $1 \times 10^{4} \mathrm{~km}$ and $1.5 \times 10^{4} \mathrm{~km}$ radius.

A relative determination of the gas-to-dust ratio for $\mathrm{G}-\mathrm{Z}$ can be made by computing the ratio of the $\mathrm{OH}$ production rate to $A f \rho$. If $\mathrm{CN}$ is produced in constant proportion to $\mathrm{OH}$ (Schleicher et al. 1987), the $Q(\mathrm{CN}) / A f \rho$ quantity is proportional to $Q(\mathrm{OH}) / A f \rho$ and, therefore, the gas-to-dust mass ratio can be determined. Since $\mathrm{G}-\mathrm{Z}$ was not imaged with the $\mathrm{OH}$ narrowband interference filter, we have used the $\mathrm{CN}$ observations and the mean ratio of $\mathrm{OH}$ to $\mathrm{CN}, \log (Q(\mathrm{OH}) / Q(\mathrm{CN}))=2.5$ (A'Hearn et al. 1995), to derive the $\mathrm{OH}$ production rate. Typical values for $\log (Q(\mathrm{OH}) / Q(\mathrm{CN}))$ range from 2.83 to 2.17 , which implies that the $\mathrm{OH}$ derived from this expression can reasonably be as much as 2 times smaller or larger than the actual value. In line with A'Hearn et al. (1995), the gas-to-dust mass ratio can be evaluated by

$\log \left(\frac{M_{\text {gas }}}{M_{\text {dust }}}\right)=\log [Q(\mathrm{OH}) / A f \rho]-25.4$.

(1)
According to this expression and using the $Q_{\mathrm{CN}}$ obtained with the Haser model (see Table 3), we computed a mean gas-todust mass ratio of $\sim 1.02$ during our observations (with an error of a factor of $\sim 2$, introduced by the approximate $\left.Q_{\mathrm{OH}}\right)$. The $\log \left(\frac{A f \rho}{Q_{\mathrm{CN}}}\right)$ on every date is also listed in Table 3 . Note that these ratios can be directly related to the log of the gas-to-dust mass ratio by simply adding 22.9 .

Since the comet was imaged in two continuum filters, we used the calibrated images to determine the average dust color and to search for spatial variations. We first computed the color two-dimensionally according to

color $=-2.5 \log \left(\frac{F_{\mathrm{c}}(\lambda)}{F_{\mathrm{c}}\left(\lambda^{\prime}\right)}\right)$,

being $F_{\mathrm{c}}$ the cometary flux in erg $\mathrm{cm}^{-2} \mathrm{~s}^{-1}$ at two different wavelengths, $\lambda$ and $\lambda^{\prime}$, with $\lambda^{\prime}>\lambda$. In our case, $\lambda$ and $\lambda^{\prime}$ correspond to the central wavelengths of the GC and RC continuum filters, i.e. $\lambda_{\mathrm{c}}=525.9$ and $\lambda_{\mathrm{c}}^{\prime}=713.3 \mathrm{~nm}$, respectively. The resulting two dimensional images show no clear features. The color or reddening of the dust can be also computed in terms of $A f \rho$ :

reddening $=\frac{1}{A f \rho} \frac{(A f \rho)^{\prime}-(A f \rho)}{\lambda^{\prime}-\lambda}$, 


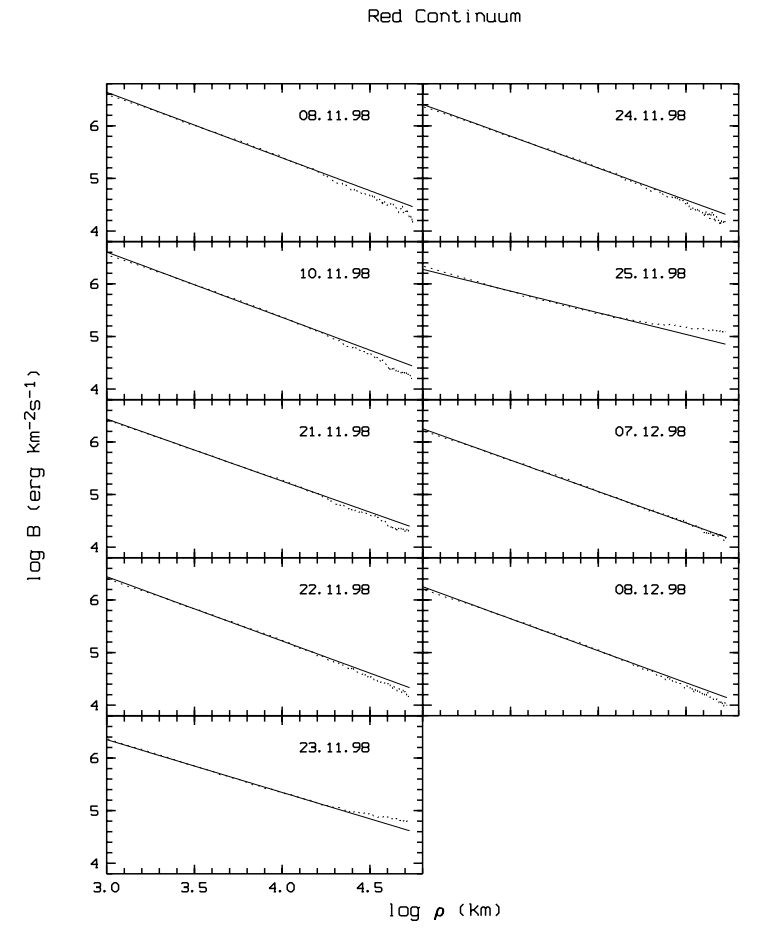

Fig. 5. Brightness profiles of Comet $21 \mathrm{P} /$ Giacobini-Zinner in the reed continuum. The profiles were obtained from azimuthal averaging of the images. The surface brightness, $B$, is plotted against the projected radius, $\rho$, in double logarithmic representation. Dots represent the observed profiles and the solid lines are the best fits verifying $\frac{\mathrm{d}(\log B)}{\mathrm{d}(\log \rho)}=-m$ starting at $\log \rho \geq 3$, with slopes listed in Table 5 .

where $(A f \rho)$ is computed at wavelengths $\lambda=525.9 \mathrm{~nm}$ and $\lambda^{\prime}=713.3 \mathrm{~nm}$.

Values for $A f \rho$ have been considered for two different circular apertures (i.e. $1 \times 10^{4}$ and $1.5 \times 10^{4} \mathrm{~km}$ ) and the average color of the dust in regions $\rho \leq 1 \times 10^{4} \mathrm{~km}$ and $\rho \leq 1.5 \times 10^{4} \mathrm{~km}$ is shown in Table 5.

\section{Discussion}

To our knowledge, no other optical observations of G-Z during the 1998 passage were made, or have been published so far. Therefore, our results can be only compared to those obtained during previous approaches of the comet to the Sun, or to global results of the gas and dust behaviour in a Jupiter-family comet at similar heliocentric distances.

As already noted by several authors (Schleicher et al. 1987; Beaver et al. 1990; Churyumov et al. 1991; Landaberry et al. 1991; Singh et al. 1997; and Ellis \& Neff 2000), it is clear that the gas production rate seems not to peak at the perihelion (Nov. 21) but some time before (see Tables 3 and 4). Although our observations all take place in the vicinity of the perihelion date, this trend is clear and the comet activity (gas and dust) steadily decreases from Nov. 8 to Dec. 8 with day-to-day variations of $\leq 20 \%$. As known from previous passages of the comet and as mentioned in the introduction, the $\mathrm{C}_{2}$ abundance, relative to $\mathrm{CN}$ (and to $\mathrm{OH}$ ), is unusually low as compared with that normally seen in comets.
During the 1985 apparition, observations by Schleicher et al. (1987) gave rise to $\log \frac{Q_{\mathrm{C}_{2}}}{Q_{\mathrm{CN}}}=-0.94$ as the average value from pre- and post-perihelion observations $\left(r_{\mathrm{H}}=1.507 \mathrm{AU}\right.$ on June 15 to $R_{\mathrm{H}}=1.457 \mathrm{AU}$, Nov. 20, 1985, perihelion date for the 1985 approach was on Sept 9). Beaver et al. (1990), from observations on June and on Sept 1985 , reported a $\mathrm{C}_{2}$ to $\mathrm{CN}$ mean relative abundance of 0.11 , that is, $\log \frac{Q_{\mathrm{C}_{2}}}{Q_{\mathrm{CN}}} \sim-0.96$. On the other hand, Landaberry et al. (1991) derived a slightly higher value of -0.52 (assuming a gas expansion velocity of $1 \mathrm{~km} \mathrm{~s}^{-1}$ for both species) from post-perihelion observations on Oct. 10, 1985 when the comet was at $r_{\mathrm{H}}=1.17 \mathrm{AU}$. Our results are closer to those of Landaberry et al. (1991) $(-0.44 \pm 0.03$ vs. -0.52$)$ which could be expectable since our and Landaberry et al.'s observations were made when the comet was closer to perihelion. From the survey of 85 comets by A'Hearn et al. (1995), G-Z was classified as a $C_{2}$ depleted comet with $\log \frac{Q_{\mathrm{C}_{2}}}{Q_{\mathrm{CN}}}=-0.67$. The $\mathrm{C}_{2}$ depletion in the coma of $\mathrm{G}-\mathrm{Z}$ has not yet been explained as the detailed chemistry giving rise to $\mathrm{C}_{2}$ is still not clear (note that the inner $\log N$ vs. $\log \rho$ profiles have been repeatedly reported to be too flat to be produced by a single parent species and in single photodissociation step). Comparing the available $\mathrm{C}_{2} \mathrm{H}_{6}$ detections in Oort-cloud comets (i.e. in comets C/1996 B2, C/1995 O1 and C/1999 H1) and Jupiter family comets (21P/GiacobiniZinner), G-Z appears to be also depleted in $\mathrm{C}_{2} \mathrm{H}_{6}$ compared to comets Hyakutake, Hale-Bopp and Lee which are "typical" comets in terms of $\mathrm{C}_{2}$ abundance.

The detection of ethane in G-Z (first detection in a Jupiterfamily comet) by Mumma et al. (2000) allowed them to establish an ethane production rate of $(7.0 \pm 1.5) \times 10^{25} \mathrm{~s}^{-1}$ at $r_{\mathrm{H}}=1.25 \mathrm{AU}$. If ethane is the only precursor of $\mathrm{C}_{2}$, and $Q_{\mathrm{C}_{2} \mathrm{H}_{6}}$ scales as $r_{\mathrm{H}}^{-2.7}$, the quantum yield of the process $\mathrm{C}_{2} \mathrm{H}_{6} \rightarrow \mathrm{C}_{2}+$ products is $\geq 0.09$, i.e. $\sim 10$ molecules of ethane are required to form one $\mathrm{C}_{2}$ molecule. From the same observations, a line of $\mathrm{H}_{2} \mathrm{O}\left(1_{11}-1^{10}\right.$ in the $v_{3}-v_{2}$ hot band, Dello Russo et al. 2000) should have appeared near the clearly detected $R 1$ line of CO. No strong line was seen and the excess of flux at the proper frequency $\left(2151.26 \mathrm{~cm}^{-1}\right)$ allowed Mumma et al. to establish an upper limit for the water production rate of $(3.2 \pm 1.7) \times 10^{28} \mathrm{~s}^{-1}$ at $r_{\mathrm{H}}=1.25 \mathrm{AU}$ (October 2-10, 1998). Scaling this production rate by $r_{\mathrm{H}}^{k}$ with $k=-2.7$ (A'Hearn et al. 1995) to $\sim 1.04 \mathrm{AU}$ at the time of our observations, the water production rate could have been $\sim 5.3 \times 10^{28} \mathrm{~s}^{-1}$. Therefore, the production rate of $\mathrm{C}_{2} \mathrm{H}_{6}$ relative to that of $\mathrm{H}_{2} \mathrm{O}$ is much higher than the $\mathrm{C}_{2}$ one $\left(2.2 \times 10^{-2}\right.$ vs. $\left.2.0 \times 10^{-4}\right)$ assuming that the variations of the gas production rates as a function of heliocentric distance are the same for both species. At this point, we note that Weaver et al. (1999) could not detect any ethane emission in the coma of $\mathrm{G}-\mathrm{Z}$ from infrared spectra taken two weeks after Mumma et al.'s observations, only an upper limit of $1.6 \times 10^{25} \mathrm{~s}^{-1}$ could be set. This upper limit to the ethane production rate together with a water production rate in the order of $(2-3) \times 10^{28}$ (Weaver et al. 1999) adequately scaled to $r_{\mathrm{H}} \sim 1.04 \mathrm{AU}$ (i.e. $2.95 \times 10^{28} \mathrm{~s}^{-1}$ ) provides $\mathrm{C}_{2} \mathrm{H}_{6} / \mathrm{H}_{2} \mathrm{O} \leq 0.05-0.08 \%$, in the same order as the $\mathrm{C}_{2} / \mathrm{H}_{2} \mathrm{O}$ derived in this work $(0.04 \%)$ when using $Q_{\mathrm{C}_{2}}=1.1 \times 10^{25} \mathrm{~s}^{-1}$ from Table 4 . 
Contrary to the $\mathrm{C}_{2}$ depletion in $\mathrm{G}-\mathrm{Z}, \mathrm{CN}$ seems to have a "quasi-normal" abundance relative to $\mathrm{H}_{2} \mathrm{O}$. The $\mathrm{CN}$ production rate from Nov. 8 to Dec. 8, 1998 slightly decreases with decreasing $r_{\mathrm{H}}$ (that is, when the comet approaches perihelion) with negligible day-to-day variations $(\sim 15 \%)$. The ratio of $\mathrm{CN}$ to $\mathrm{OH}$ production rates is $\sim 7 \times 10^{-4}$ assuming that the water production rate at $r_{\mathrm{H}}=1.1 \mathrm{AU}$ is $(5.6 \pm 0.4) \times 10^{28} \mathrm{~s}^{-1}$ (Biver et al. 1999a) conveniently scaled by $r_{\mathrm{H}}^{-2.7}$ to $\sim 1.04 \mathrm{AU}$. On the other hand, if we consider the $\mathrm{H}_{2} \mathrm{O}$ production rate given by Weaver et al. (1999) from IR observations, this ratio increases to $0.15 \%$. These two values bracket a value of $\log \frac{Q_{\mathrm{CN}}}{Q_{\mathrm{OH}}}$ between -3.15 and -2.82 , which is more characteristic of "depleted" comets (see Table VI in A'Hearn et al. 1995) as G-Z indeed is, than of a normal comet. Comparing this ratio to that obtained from previous passages of the comet, Schleicher et al. (1987), from observations at similar $r_{\mathrm{H}}$ as ours (from 1.053 to $1.043 \mathrm{AU}$ ), derived a $\mathrm{CN}$ production rate of $7.2 \times 10^{25} \leq Q_{\mathrm{CN}} \leq 3.63 \times 10^{25} \mathrm{~s}^{-1}$ by means of the Haser model. The values presented here are similar to these ones, whereas the relative abundance of $\mathrm{CN}$ to $\mathrm{OH}$ during the 1985 passage $(0.2 \%)$ can be considered similar to the one derived in this work during the 1998 approach $(0.15$ $0.07 \%$ ). These differences, if any, given the uncertainties in the determination of $\mathrm{OH}$ production, can be attributed to different behaviour of the comet and/or to different scale lengths in the Haser modeling, and/or to different production rates of $\mathrm{H}_{2} \mathrm{O}$ directly measured in the IR or indirectly $(\mathrm{OH}$ in radio wavelengths or in the UV).

During their IR investigation of volatiles in the coma of G-Z, Weaver et al. (1999) were not able to detect HCN. This non-detection allowed them to set an upper limit for the HCN production rate of $5.4 \times 10^{25} \mathrm{~s}^{-1}$, later confirmed by Biver et al. (1999a) $\left(Q_{\mathrm{HCN}} \sim 3 \times 10^{25} \mathrm{~s}^{-1}\right)$, and a quotient of the $\mathrm{HCN}$ to $\mathrm{H}_{2} \mathrm{O}$ abundance of 0.05 to $0.07 \%$ at $r_{\mathrm{H}} \sim 1.1 \mathrm{AU}$ below the normal abundances of $0.08-0.25 \%$ (Biver et al. 2002). It is interesting to note that this $\mathrm{HCN} / \mathrm{H}_{2} \mathrm{O}$ is very similar to the $\mathrm{CN} / \mathrm{H}_{2} \mathrm{O}(=0.11 \%$ to $0.22 \%)$ that we report from our $\mathrm{CN}$ measurements and available water measurement, either from IR (Weaver et al. 1999) or OH radio observations (Biver et al. 1999a). At first sight, this result seems to indicate that $\mathrm{CN}$ can be solely produced by $\mathrm{HCN}$, however the required $\tau_{\mathrm{p}}\left(\sim 2 \times 10^{4} \mathrm{~s}\right)$ to fit the $\mathrm{CN}$ column density profiles by means of the Vectorial model is low when compared with determinations by Bockelée-Morvan \& Crovisier (1985) or by Huebner \& Link (1999). The nowadays available information is not enough to rule out other $\mathrm{CN}$ parent, species such as $\mathrm{C}_{2} \mathrm{~N}_{2}$ (Festou et al. 1998; Komitov \& Bonev 1999), $\mathrm{C}_{4} \mathrm{~N}_{2}+\mathrm{HC}_{3} \mathrm{~N}$ (Krasnopolsky $1991)$ and/or evaporation from the dust grains.

Our dust analysis of the surface brightness profiles has shown that most profiles can be fit by the $\operatorname{law} \log B \sim$ $-m \log \rho$ with $1<m \leq 1.33$ (except the red continuum profile on Nov. 25 which shows a slightly flatter profile) in contradiction with previous analysis of the $\mathrm{G}-\mathrm{Z}$ brightness profiles by Jewitt \& Meech (1987). These authors indicated that the $\log B$ profile vs. $\log \rho$ for $\mathrm{G}-\mathrm{Z}$ did not strictly follow the law $\log B \sim-m \log \rho$ with $1 \leq m \leq 3 / 2$ at distances larger than $10^{\prime \prime}$ from the nucleus $(\sim 7500 \mathrm{~km}$ or $\log \rho=2.87$ in our case), and the negative curvature $\mathrm{d} m / \mathrm{d} \log \rho<0$ could be indicative of variations in the strength of the nucleus source on time scales comparable to the grain flight time. In fact, the activity of G-Z did vary during our observations, but the derived continuum profiles do not exhibit slopes higher than $3 / 2$ at distances from the nucleus larger than $10^{\prime \prime}(\log \rho \sim 2.9)$. The dust production rate, either derived from the green or the red continuum filter, decreases from Nov. 8 to Dec. 8, regardless of the approach of the comet to the Sun.

The polarization of the scattered light of the dust particles in G-Z has an unusual behaviour. Contrary to all other comets, the wavelength gradient of the polarization of the continuum is negative. Kiselev et al. (2000a,b) have attributed this effect to a high abundance of organic matter in its dust and/or to an overabundance of large particles, also supported by the weak $10 \mu \mathrm{m}$ silicate feature detected by Hanner et al. (1992). However, comets with low abundance of submicron silicate grains have a low degree of polarization (10-15\%) for both the blue and the red domain of the spectrum at large phase angles, this not being the case for G-Z. Due to this, Kiselev et al. (2000a,b) favour the idea that the dust particles in $\mathrm{G}-\mathrm{Z}$ have an overabundance of organic matter. Furthermore, an abundance of large particles in the coma may also produce a redder colour as is computed from our continuum images at $\rho \leq 10000 \mathrm{~km}$. The reddening of the dust ranges from a common 13\%/1000 $\AA$ (in agreement with the $\sim 15 \%$ reported by Schleicher et al. 1987) to a high value of $29 \% / 1000 \AA$ which could be indicative of an overabundance of large grains in the dust coma of $\mathrm{G}-\mathrm{Z}$ after the perihelion (Dec. 7 and 8, 1998).

Although the general behaviour of the dust color is to be redder than the Sun, there are two dates when this trend was not followed. On Nov. 10, the dust was neutral (Sun color) and on Nov. 25 it was much bluer than the Sun. On the latter date, the dust production rate decreased by a $20 \%$ (if measured in the green continuum) and by a $50 \%$ (if measured in the red continuum) with respect to the previous day, and this variation had no clear counterpart in the gas activity since it was steadily decreasing from Nov. 8 to Dec. 8. Previous observations by Singh et al. (1997) also noticed a change of color (from red to blue relative to the Sun) of grains during a $24 \mathrm{~h}$ period in $\mathrm{G}-\mathrm{Z}$ together with a $Q_{\mathrm{CN}}$ decrease of $25 \%$. However, as these authors only observed the comet on Oct. 15 and 16, 1985, we cannot conclude that this sudden blueing of the dust was due to a noticeable variation in the cometary activity.

Regarding the gas-to-dust mass ratio, it can vary largely depending on the water production rate we use (from 1.02 to 3.6, lower than and similar to previous passages, respectively). Schleicher et al. (1987) determined a relative gas-todust ratio by simply dividing the $\mathrm{OH}$ production rate $\left(\mathrm{s}^{-1}\right)$ and Af $\rho(\mathrm{cm})$ which resulted in a value of $1 \times 10^{26} \mathrm{~s}^{-1} \mathrm{~cm}^{-1}$. By applying Eq. (1), the gas-to-mass ratio is $\sim 4$. Singh et al. (1997) concluded that the dust-to-gas ratio was always less than unity (that is, gas-to-dust ratio > 1) during Oct. 15-16, 1985. In Table III in A'Hearn et al. (1995), $\log \frac{A f \rho}{Q_{\mathrm{OH}}}$ is -25.94 , which means a gas-to-dust mass ratio of $\sim 3.5$. Mumma et al.'s (2000) determination for the water production rate at $r_{\mathrm{H}}=1.25 \mathrm{AU}$ adequately scaled to $\sim 1.04 \mathrm{AU}$, at the time of our observations, allows us to derive a mean gas-to-dust mass ratio of 3.6 from Nov. 8 to Dec. 8, 1998. Let us note that this value might be affected by some errors since the gas production can substantially 
vary from one comet to another and the expression $Q \sim r_{\mathrm{H}}^{-2.7}$ may be inaccurate for $\mathrm{G}-\mathrm{Z}$. In fact, if we assume this water production rate as valid, this would mean that $\mathrm{OH}$ is produced at a rate of $3.51 \times 10^{28} \mathrm{~s}^{-1}$ (relatively higher than the maximum reported measurement of $3.23 \times 10^{28} \mathrm{~s}^{-1}$ in A'Hearn et al. 1995), meaning $\log (Q(\mathrm{OH}) / Q(\mathrm{CN}))=3.1$, larger than the typical 2.83-2.17 given by A'Hearn et al. (1995) or than the 2.74 (specific for $\mathrm{G}-\mathrm{Z}$ ) listed in their Table III. All these values are not associated with an unusually low abundance of $\mathrm{C}_{2}, \mathrm{C}_{3}$, $\mathrm{NH}$, and $\mathrm{NH}_{2}$, that is, regarding the gas-to-dust ratio, $\mathrm{G}-\mathrm{Z}$ is a typical comet.

Acknowledgements. V. M. acknowledges the support of a MUTIS Fellowship from the Agencia Española de Cooperación Internacional (AECI). This research has been partially supported by the Spanish Ministerio de Ciencia y Tecnología under contract PNE-002/2000-C. We are very grateful to J. Crovisier for his constructive comments which have greatly improved the paper.

\section{References}

A'Hearn, M. F., Schleicher, D. G., Feldmann, P. D., Millis, R. L., \& Thompson, D. T. 1984, AJ, 89, 579

A'Hearn, M. F., Birch, P. V., Feldmann, P. D., \& Millis, R. L. 1985, Icarus, 64, 1

A'Hearn, M. F., Millis, R. L., Schleicher, D. G., Osip, D. J., \& Birch, P. V. 1995, Icarus, 118, 223

Beaver, J. E., Wagner, R. M., Schleicher, D. G., \& Lutz, B. L. 1990, ApJ, 360, 696

Biver, N., Crovisier, J., Davies, J. K., et al. 1999a, in abstract book for Asteroids, Comets and Meteors, 26-30 July 1999a, Cornell University, Ithaca

Biver, N., Bockelée-Morvan, D., Crovisier, J., et al. 1999b, AJ, 118, 1850

Biver, N., Bockelée-Morvan, D., Crovisier, J., et al. 2000, AJ, 120, 1554

Biver, N. D., Bockelée-Morvan, J. Crovisier, P., et al. 2002, Earth, Moon \& Planets, Proc. IAU Coll., 186, in press

Bobrovnikoff, N. T. 1927, ApJ, 66, 439

Bockelée Morvan, D. 1997, in Molecules in Astrophysics: Probes and Processes, ed. E. F. Van Dishoek (Kluwer Academic, Dordrecht), pp. 219-235

Bockelée-Morvan, D., \& Crovisier, J. 1985, A\&A, 151, 90

Churyumov, K. I., \& Rosenbush, V. K. 1991, Astron. Nachr., 312, 385
Cochran, A. L., \& Barker, E. S. 1987, AJ, 93, 239

Combi, M. R., \& Delsemme, A. H. 1980a, ApJ, 237, 633

Combi, M. R., \& Delsemme, A. H. 1980b, ApJ, 237, 641

Dello Russo, N., Mumma, M. J., DiSanti, J. A., et al. 2000, Icarus, 143,324

Ellis, T. A., \& Neff, J. S. 2000, Icarus, 145, 591

Farnham, T., Schleicher, D., \& A'Hearn, M. F. 2000, Icarus, 147, 180

Festou, M. C. 1981, A\&A, 95, 69

Festou, M. C., Barale, O., Davidge, T., et al. 1998, BAAS, 30, 1089

Hanner, M. S., Veeder, G. J., \& Tokunaga, A. T. 1992, AJ, 104, 386

Haser, L. 1957, Bull. Cl. Sci. Acad. R. Belg., 43, 740

Herbig, G. H. 1976, in The Study of Comets, ed. B. Donn, M. Mumma, W. Jackson, \& M. F. A'Hearn, NASA SP-393, 136

Huebner, W. F., \& Link, R. 1999, http://espsun. space. swri.edu/spacephysics/atomic/ html/photoabs.htm

Huebner, W. F., Keady, J. J., \& Lyon, S. P. 1992, Astrophys. Space Sci., 195, 1

Jewitt, D. C., \& Meech, K. J. 1987, ApJ, 317, 992

Kiselev, N. N., Jockers, K., Rosenbush, V. K., et al. 2000a, Planet. Space Sci., 48, 1005

Kiselev, N. N., Jockers, K., \& Rosenbush, V. K. 2000b, EMP 82-83, 141

Komitov, B., \& Bonev, T. 1999, BAAS, 31, 1127

Konno, I., \& Wyckoff, S. 1989, Adv. Space Res., 9, 163

Krasnopolsky, V. A. 1991, A\&A, 245, 310

Landaberry, S. J. C., Singh, P. D., \& de Freitas Pacheco, J. A. 1991, A\&A, 246, 597

Lebedinets, V. N. 1991, Sol. Sys. Res., 25, 49

McFadden, L. A., A'Hearn, M. F., Fledman, P. D., et al. 1987, Icarus, 69, 329

Mumma, M. J., DiSanti, M. A., Dello Russo, N., Magee-Sauter, K., \& Rettig, T. W. 2000, ApJ, 531, L155

Randall, C. E., Schleicher, D. G., Ballou, R. G., \& Osip, D. J. 1992, BAAS, 24, 1002

Schleicher, D. G. 1983, Ph. D. Dissertation, Univ. of Maryland

Schleicher, D. G., Millis, R. L., \& Birch, P. V. 1987, A\&A, 187, 531

Schulz, R., A'Hearn, M. F., Birch, P. V., et al. 1993, Icarus, 104, 206

Schulz, R., McFadden, L. A., Chamberlin, A. B., A'Hearn, M. F., \& Schleicher, D. G. 1994, Icarus, 190, 145

Singh, P. D., Huebner, W. F., Costa, R. D. D., Landaberry, S. J. C., \& de Freitas Pacheo, J. A. 1997, Planet. Space Sci., 45, 455

Weaver, H. A., Chin, G., Bockelée-Morvan, D., et al. 1999, Icarus, 142,482 